\title{
Study of Volume Restriction Methods Based on the Melody Structure of Music for Hearing Protection
}

\author{
Daiki Okabe ${ }^{\mathrm{a}}$, Yoshihisa Nakatoh, ${ }^{\mathrm{a}, *}$ \\ ${ }^{a}$ Kyushu Institute of Technology, 1-1 Sensui-cho Tobata-ku Kitakyusyu 804-8550, City and Postcode, Japan \\ ${ }^{\mathrm{b}} \mathrm{Second}$ affiliation, Address, City and Postcode, Country \\ *nakatoh@ecs.kyutech.ac.jp
}

\begin{abstract}
In this paper, in order to prevent hearing loss from music listening using a portable music player, the possibility that lower the volume to a safe level without be noticed by the user (difference of perceived attenuating level) are evaluated. As a result, the notice happened when volume level was attenuated $12 \pm 4 \mathrm{~dB}$, and it not be affected by changing attenuation rate of volume level. Based on the above experiments, we examine the melody structure of music by attenuate the volume separately for melody structure (Intro part, Verse, Chorus, Interlude). As a result, it was found that the intro part was hard to notice the volume attenuation, and in the part of the chorus, it was easy to notice the volume attenuation. Next, we evaluated difference of the perceived attenuating level considering frequency characteristics. As a result for the low frequency, it was difficult to notice the volume attenuation, and the high frequency tends to notice the volume attenuation.
\end{abstract}

Keywords: headphone hearing loss, portable music player, hearing protection, music components, frequency.

\section{Introduction}

In recent years, portable music players have spread because of the convenience of enjoying music easily. On the other hand, it is reported that a risk of causing hearing loss is increased by listening to music at loud volume and for a long time ${ }^{(1,2,3)}$. In fact, WHO and EU have announced an indicator for listening to music safely. WHO showed people listening to music at $85 \mathrm{~dB}$ per day for one hour or more is dangerous. EU showed people listening to music at $80 \mathrm{~dB}$ per day for 5.7 hours or more is dangerous. Therefore, we propose a method to prevent hearing loss by lowering the volume so as not to notice when the user is listening to music at dangerous volume. Higashi et al. ${ }^{(4)}$, it measured the optimal listening level in order to understand how loudly the user (young, elderly people) listens to music on portable music player. More it measured difference of perceived attenuating level as well as reaction time until the user notices when sound volume was gradually turned as well as reaction level and the reaction time were measured. The subjects consisted of seven younger individuals ranging from 22 years old to 25 years old (6 males, 1 female), and five elderly individuals from 72 years old 77 years old (all male). The young people had normal hearing, and the elderly confirmed that they have moderate hearing loss to mild hearing loss. In the experiment of measuring the optimal listening level, they used 6 songs in total, including Western rock and pop music, Japanese rock, punk and pop music, and Enka. They used 4 background noises in total, including in the bus, in the station premises, noisy load, in the subway, and no background noise. As a result, most adults and some elderly people had the optimal listening level that exceeded the dangerous sound pressure level of $80 \mathrm{~dB}$ pointed out by the EU. Therefore, it was known that there was a need to lower the volume when listening to music at a level causing hearing loss.

In the measurement of the difference of perceived attenuating level against the volume attenuation, the subjects and the music consisted of the same people in the measurement experiment of the optimum listening level. The background noise is taken from a real station because of the power spectrum is available in the whole frequency range. And these music were set with the equivalent noise 
level of $85 \mathrm{~dB}$ as the initial value, and then the attenuation of these music were started from a certain point of these music. The ways to lower the volume were set to four kinds in total of $0.5,0.3,0.25,0.2[\mathrm{~dB} / \mathrm{sec}]$. As a result, it was known that at each attenuation rate, adults perceived in the range of about $12 \mathrm{~dB} \pm 4 \mathrm{~dB}$ from the start of the volume attenuation, and the elderly perceived in the range of about $13 \mathrm{~dB} \pm 4 \mathrm{~dB}$ from the start of the volume attenuation.

In this study, we first evaluate the difference of perceived attenuating level at a faster attenuation rate than the previous study. Also, we evaluate the difference of perceived attenuating level when the volume is attenuated for each structure of Intro part, Verse, Chorus, etc. Based on these results, we evaluate the difference of perceived attenuating level when gradually attenuating the sound volume for each frequency using the $1 / 1$ octave band pure tone and band noise.

\section{Evaluation of the difference of perceived attenuating level in relation to attenuation rate}

In this chapter, we evaluate how much the subjects noticed the volume attenuation when the volume level of the music is gradually attenuated..

\subsection{The proposed method}

Experimental results of the previous study shows little difference in the difference of perceived attenuating level due to attenuation rate. Therefore, we conduct the experiment to confirm whether the same result would result at the attenuation rates faster than the attenuation rates of the previous study. The ways to lower the volume are set to five kinds in total of $0.5,0.67,1.0,2.0,4.0[\mathrm{~dB} / \mathrm{sec}]$, and we use 3 songs in total, including western pop music, Japanese rock and punk music. Fig. 1 shows how the sound source attenuates. The presented music contained the music whose volume gradually increases or did not change as a dummy. We created a smartphone application for evaluation and played these music through the headphone (SENNHEISER HD205) connected to Apple's iPod touch with the application. In addition, the experiment was conducted in the soundproof chamber, and under the background noise in the station $(66.2 \mathrm{~dB})$ considering the situation of actually listening to the music. The subjects were 5 adults (men) with normal hearing from 21 to 23 years old.

\subsection{The experimental result}

Fig. 2 shows the average value and standard deviation of the difference of perceived attenuating level for five kinds of the attenuation rates. From Fig. 2, it was found that the subjects were aware of the volume attenuation in the range of about $11 \mathrm{~dB} \pm 3 \mathrm{~dB}$ after the volume attenuation was started.

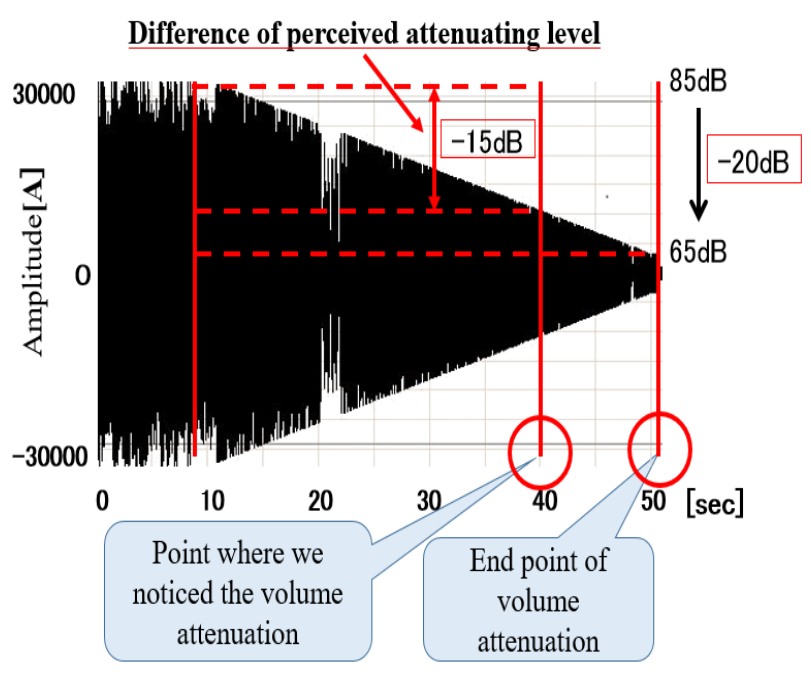

Fig1. Waveform attenuation

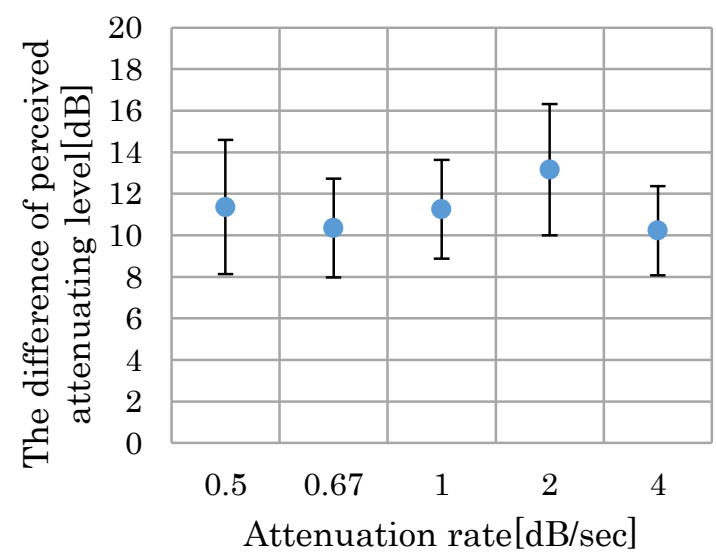

Fig2.The difference of perceived attenuating level in relation to attenuation rate

According to the questionnaire after the experiment, there were many opinions that the attenuation rate of $2.0 \mathrm{~dB}$ / sec attenuated by $20 \mathrm{~dB}$ in 10 seconds, so the attenuation was fast and it was hard to notice. Therefore, it seems that it 
was delayed that the subjects noticed the attenuation more than other attenuation rates. Also, the attenuation rate of 4.0 $\mathrm{dB} / \mathrm{sec}$ in a very short time of 5 seconds, it was predicted that it would end at $20 \mathrm{~dB}$ downward when it noticed the attenuation, but expected to perceive it in the vicinity, but the result was different from our expectation. According to the result of the questionnaire, it was thought that this was due to the fact that it instantaneously became aware of immediately because the sound volume decreased with a large amount of attenuation. If the attenuation rate is further increased, it is thought that even if we perceive the attenuation, since the decay time is extremely short, it will result in a lot of uncertainty, such as being drastically attenuated around the time of stoppage. Therefore, we found that there was no significant difference in the difference of perceived attenuating level even at a faster attenuation rate than in the previous study. On the other hand, there was hardly any difference in the difference of perceived attenuating level depending on the type of music.

\section{Evaluation of the difference of perceived attenuating level in relation to music components}

In this chapter, we evaluate the influence of the music components on the difference of perceived attenuating level.

\subsection{The proposed method}

Subjects and experimental conditions were the same as those in the previous chapter. For the presentation music in this experiment, we edited so that attenuation starts from 4 places of Intro part, Verse, Chorus and Interlude in the three kinds of music of chapter 2 , and made it possible to compare the difference of perceived attenuating level for each structure of music. For this reason, the attenuation rate was set at $0.5 \mathrm{~dB} / \mathrm{sec}$ all. Therefore, it was set to be 4 experimental sound sources with different attenuation points per song.

\subsection{The experimental result}

Fig. 3 shows the average value and the standard deviation of the difference of perceived attenuating level with respect to the attenuation starting point. Since the accompaniment part had no lyrics and it was a structure with comparatively little change in the structure of music, we anticipated that attenuation may not be noticed, and the expected results were obtained. On the other hand, the part of chorus was predictable of attenuation because there was undulation in the tune, and expectation was obtained although there were some individual differences. Since many similar opinions were obtained from the questionnaire after the experiment, it seemed that this result was obtained. Therefore, the presence or absence of lyrics and the undulation of the tune may affect the attenuation perception level difference. Both Verse and Interlude were of the same degree of the difference of perceived attenuating level. It was considered that Verse is basically not a structure with intense change. Since the Interlude was after the change in tune especially after it was changed, it seemed as if the sound pressure had decreased as a whole, but this was a basic structure in the structure of music, it is natural. Therefore, it seemed that it was a characteristic result because it was not misunderstood that the sound pressure fell immediately by the interlude. From these facts, it seems that Verse and Interlude were the same level of the difference of perceived attenuating level.

\section{Evaluation of the difference of perceived attenuating level in relation to frequency}

In the experiments up to the previous chapter, the details are unclear as to what factors in music affected the difference of perceived attenuating level. Therefore, we focus on frequency characteristic. 


\subsection{The proposed method}

Subjects and experimental conditions were the same as those in the previous chapter. The presentation sounds in this experiment are divided $0 \mathrm{~Hz}$ to $8 \mathrm{kHz}$ in the octave band and created a pure tone for each center frequency, they are total of 8 kinds of pure tones, $1 / 1$ octave band $(62.5 \mathrm{~Hz}$ to $8000 \mathrm{~Hz}$ ). The attenuation rate is unified to $0.5 \mathrm{~dB} / \mathrm{sec}$. Considering the A characteristic, when the pure tone of 1 $\mathrm{kHz}$ is set to $85 \mathrm{dBA}$, the power correction is performed so as to be $85 \mathrm{dBA}$ also at the other frequency.

\subsection{The experimental result}

Fig. 4 shows the average value of the difference of perceived attenuating level for each frequency of pure tone. Subjects were more likely to notice the attenuation of the volume than pure tones compared to the music of the previous chapter. In addition, subjects were more likely to notice the attenuation at higher frequencies for both pure tones, and tend to be less aware of attenuation at lower frequencies. According to the questionnaire after the experiment, there are opinions that "The pure tone is more apt to notice the attenuation than the music." or "opinion

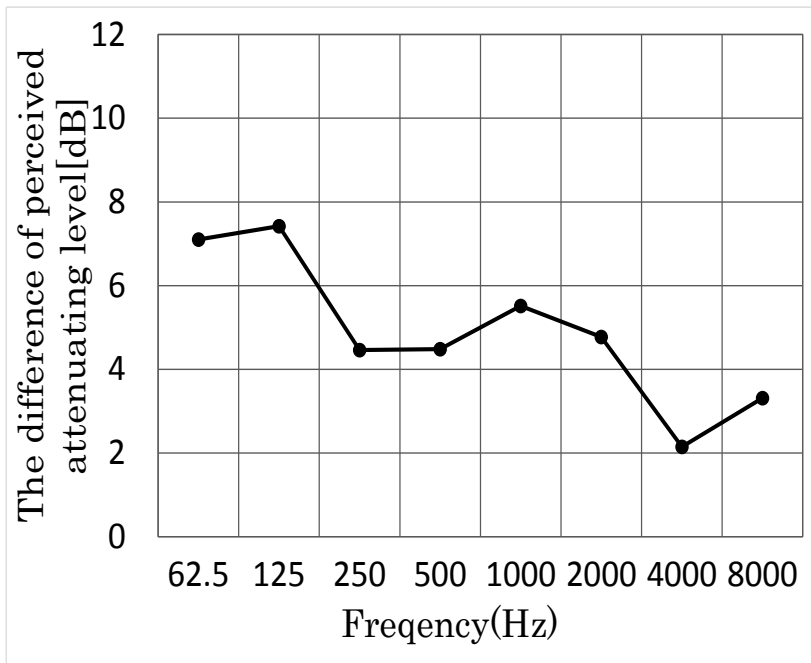

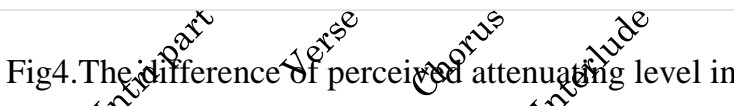
rełation to the frequency of pure tone

Fig3.The difference of perceived attenuating level in relation to the music components that it is hard to tell if the volume of the low frequency lowers", which is consistent with the experimental result.

\section{Conclusion}

In this study, we evaluated the difference of perceived attenuating level when the volume was gradually attenuated, and it was found that the difference of perceived attenuating level was less influenced by the type of music, but it was found that the difference of perceived attenuating level changes depending on the melody structure of music. Moreover, regarding the evaluation of the attenuation perception level difference for each frequency, it is difficult to notice the low frequency volume attenuation, and the high frequency tends to be noticed easily. In the future, we plan to study the volume attenuation method which is hard to be noticed by the user, and make an useful application for users.

\section{Acknowledgment}

Part of this study, was conducted with the assistance of the Ministry of Education Science and Research Grants Fund grant (NO.24500664).

\section{References}

(1) Fumitaka Saito, Yoiti Suzuki, and Heli Laitinen, "Consideration concerning hearing of amount of exposure of sound by music and young people's hearing ", The Journal of The Acoustical Society of Japan Vol. 63 No. 4 pp233-238 (2007)

(2) Mariko Hamamura, Naoki Kishigami and Shinichiro Iwamiya, "Difference in optimum listening level for reproduced music between men and women",Journal of the Acoustical Society of Japan Vol. 70 No. 10 pp.525 -533 (2015)

(3) Mariko Hamamura, Manami Aono and Shinichiro Iwamiya, "Differences in the preferred listening levels of ambient music, sound signs, public announcements, and natural environmental sounds between men and women", Journal of the Acoustical Society of Japan Vol. 71 No. 2 pp.65 -72 (2015)

(4) Teruhisa Higashi, Yuto Tanaka, and Yoshihisa Nakatoh 
"Study of Volume Limit for Hearing Protection in the Music Listening" IIAE CONFERENCE SYSTEM, The 3rd IIAE International Conference on Intelligent Systems and Image Processing 2015 (ICISIP2015) 